\title{
Traslados entre EPS en Colombia: ¿Qué dicen las historias laborales de cotizantes en cinco ciudades del país?*
}

\section{Transfers among Health Plans in Colombia: What do Government Enrollment Files Say in Five Cities across the Country?}

\section{Transferências entre EPS na Colômbia: 0 que as histórias laborais de beneficiários em cinco cidades do país dizem}

Fecha de recepción: 05-05-15 Fecha de aceptación: 17-02-16 Disponible en línea: 03-05-16

doi:10.11144/Javeriana.rgyps15-30.tecq

Cómo citar este artículo:

Prada-Ríos SI. Traslados entre EPS en Colombia: ¿Qué dicen las historias laborales de cotizantes en cinco ciudades del país? Rev. Gerenc. Polít. Salud. 2016; 15(30): 176-192. http://dx.doi.org/10.11144/Javeriana.rgyps15-30.tecq

Artículo de investigación. Este trabajo no ha sido presentado en ningún evento, ni ha sido financiado por ninguna entidad. El autor agradece la valiosa asistencia de José Ricardo Martínez, María José Ochoa, Andrés Aguirre y Mayra Alejandra Sarria. Las opiniones y posibles errores son de responsabilidad exclusiva del autor y no comprometen a la institución para la que trabaja.

176 Ph.D. en Política Pública, University of Maryland. Investigador Senior Proesa y profesor, Universidad Icesi, Facultad de Ciencias Administrativas y Económicas, Departamento de Economía, Calle 18 no 122-135, Cali, Colombia. Correo electrónico: siprada@proesa.org.co. 


\section{Resumen}

Un argumento utilizado por las EPS para no invertir en promoción y prevención, es la posibilidad de que dicha inversión se pierda porque el afiliado se puede trasladar a otra EPS. A la vez, la libre elección de EPS es pilar fundamental del sistema de salud, en tanto que permite a los afiliados dejar las EPs de mala calidad y trasladarse a aquellas de buena calidad. Este estudio brinda información cuantitativa sobre el tamaño del fenómeno de traslados entre EPS, con el fin de dar luces sobre este debate. Para ello se usó información pública sobre las historias de afiliación de los últimos catorce años (2001-2014), para una muestra aleatoria de adultos entre cuarenta y cincuenta años en cinco ciudades capitales del país. Se calcularon tasas de traslado en periodos de afiliación continua. Se encontró que más del $56 \%$ de los cotizantes tuvieron cuatro o más periodos de cotización; dentro de un mismo periodo de cotización el porcentaje de las personas que no cambió de EPS fue siempre superior al 54\%; en un año calendario, menos del $6 \%$ decidió trasladarse; existía una correlación débil entre traslados e indicadores de calidad de servicio. Los resultados muestran que la tasa de traslados entre EPS es baja, pero que existe una alta fragmentación en los periodos de cotización de una persona. La gente, en consecuencia, no se traslada, sino que interrumpe su afiliación, lo que permite concluir que este comportamiento está marcado por las fluctuaciones del mercado laboral. El traslado de EPS como mecanismo de rendición de cuentas no es un fenómeno frecuente, y si el Gobierno quiere que funcione debe ser rediseñado.

Palabras clave: sistemas de salud; Colombia; comportamiento del consumidor; conducta de elección; libre elección del paciente; seguro de salud

\section{Abstract}

Insurers (нмоз) argue that there is no incentive to invest in prevention activities because members can easily move to another insurance company. At the same time freedom to choose нмоs is a key accountability mechanism of the Colombian Health System because citizens can leave low-quality insurers and enroll in high-quality insurers. This paper quantifies the size of the rate of transfers between HMOs in the country to shed light on this debate. This study used publicly available information on affiliation histories to the health system for the last 14 years (2001-2014) for a random sample of adults between 40 and 50 in five different capital cities. The rate of enrollee transfers within a continuous enrollment period between нмо was estimated. Results showed that $56 \%$ of enrollees had four or more enrollment periods; within the same enrollment period at least $54 \%$ of enrollees did not transfer between нмо; in a year less than $6 \%$ decided to transfer; there was a weak correlation between transfers and нмо service quality indicators. Results showed that the transfer rate is low but that the drop-out rate is high. People do not transfer but leaves the systems frequently, which suggest that this behavior is explained by labor market fluctuations. The transfer between нмо is not frequent and this suggests that as accountability mechanism is not working and thus needs to be redesigned.

Keywords: health systems; Colombia; consumer behavior; choice behavior; patient freedom of choice laws; health insurance

\section{Resumo}

Uma argumentação utilizada pelas EPS para não investir em promoção e prevenção é a possibilidade de tal investimento se perder pelo afiliado se transferir para outra EPS. Ao mesmo tempo, a livre eleição de EPS é pilar fundamental do sistema de saúde, em tanto que permite os beneficiários deixar as EPs de qualidade ruim e se transferir para aquelas de boa qualidade. Este estudo fornece informação quantitativa sobre o comprimento do fenómeno de transferências entre EPS, A fim de dar luzes sobre este debate. Para isso utilizou-se informação pública sobre as histórias de transferências dos últimos 14 anos (2001-2014), para uma amostra aleatória de adultos entre 40 e 50 anos em cinco cidades capitais do país. Calcularam-se taxas de transferência em períodos de afiliação contínua. Encontrou-se que mais do que $56 \%$ dos contribuintes tiveram quatro ou mais períodos de contribuição; dentro do mesmo período de contribuição a porcentagem das pessoas que não mudaram de EPS foi sempre superior a 54\%; em um ano, menos de $6 \%$ decidiu se transferir; havia uma correlação fraca entre transferências e indicadores de qualidade de serviço. Os resultados mostram que a taxa de transferências entre EPs é baixa, mais houve elevada fragmentação nos períodos de contribuição de uma pessoa. O pessoal, em consequência, não se transfere, senão que interrompe sua afiliação, o que permite concluir que tal comportamento está marcado por flutuações no mercado de trabalho. A transferência de EPS como mecanismo de rendição de contas não é uma ocorrência frequente, e se o Governo quiser que ela funcione deve ser redesenhado.

Palavras-chave: sistemas de saúde; Colômbia; comportamento do consumidor; comportamento de escolha; livre eleição do paciente; seguro de saúde 


\section{Introducción}

La legislación colombiana exige que todo ciudadano esté afiliado a una Entidad Promotora de salud (EPS) del Sistema General de Seguridad Social en Salud (sGsss). En el régimen contributivo el afiliado contribuye con un porcentaje de su ingreso, en el régimen subsidiado el Gobierno hace la contribución a nombre del afiliado. En ausencia del mecanismo de precios como forma de ajuste entre oferta y demanda en el mercado del aseguramiento, el sistema permite a los individuos escoger libremente la EPS y trasladarse entre ellas, bajo condiciones definidas por ley.

Sin pérdida de generalidad, el traslado a otra EPS es posible después de cumplir un año de afiliación (Ley 1122 del 2007), definido como doce meses de afiliación continua y con pagos de cotización al día. Después de pasado este periodo, siempre y cuando el afiliado no esté recibiendo tratamiento por enfermedades de alto costo, y no se encuentre en mora, puede efectuar el traslado a otra EPS. En el papel el costo de transacción es bajo, ya que solo implica diligenciar el formulario de afiliación con la nueva EPS, y es dicha EPS receptora la que se encarga del resto del trámite. No obstante, dependiendo de las prácticas administrativas de ambas EPS, el trámite puede resultar costoso. La EPS que recibe la solicitud de nuevo afiliado puede rechazarlo si comprueba que este es un paciente que está recibiendo tratamiento de alto costo. Las restricciones a la movilidad de pacientes de alto costo operan como mecanismo para evitar la concentración de riesgo en una sola EPS.

La libre elección después del primer año de permanencia es parte del diseño del sistema y opera como el mecanismo de mercado para que los afiliados premien o castiguen

178 a las EPs, de tal forma que solo aquellas con resultados sobrevivan en el mercado. En la literatura esto se denomina la ruta corta de la rendición de cuentas (1). La posibilidad de trasladarse entre EPS ha sido un argumento sugerido como desincentivo para que estas inviertan en actividades de promoción y prevención entre sus afiliados.

En Colombia no existe una entidad o un observatorio que de manera sistemática estudie las cifras de traslados entre EPS y las razones para ello. En el régimen contributivo (RC), sin embargo, la Asociación Colombiana de Empresas de Medicina Integral (Acemi) ha construido el Sistema de Información de Afiliados y de Movilidad (SIAM), como un servicio operativo para que las EPs puedan consultar la historia de afiliación tanto de cotizantes como de beneficiarios, incluidos la historia de traslados. Este sistema no es abierto al público y no presenta resultados agregados.

De acuerdo con una EPS del RC que permitió acceso a sus datos, en el año 2013 el 3,7\% de los afiliados pidieron traslado a otras EPS; de estas solicitudes, el $62 \%$ fue aprobado y el restante $38 \%$ rechazado. En el 2014, el porcentaje de peticiones de traslados aumentó a 5,3\%, de los cuales $52 \%$ fueron rechazadas y $48 \%$ aprobadas. De forma que el número efectivo de traslados fue de $2,3 \%$ y de 2,8\% para los dos años, respectivamente. En cuanto a traslados de otras EPS a la misma EPs, tomando como denominador el mismo número de afiliados, se encuentra que pidieron traslado a esta EPS el $2,8 \%$ en el 2013 y el 7,2\% en el 2014. De estos efectivamente fueron aprobados el 53 y el $32 \%$, respectivamente.

Por otro lado, de acuerdo con la Encuesta de Evaluación de Servicios de EPs 2013 del Ministerio de Salud y Protección Social, es posible establecer la intención de los usuarios de cambiarse a otra EPS (2). Los resultados se presentan en la tabla 1. Según estos, aproximadamente el $16 \%$ de los usuarios del 
régimen contributivo ha pensado en cambiarse en alguna oportunidad de EPS, siendo la demora en la prestación de servicios la razón que más peso tiene, con un $30,8 \%$.

Los resultados de traslados de la EPS mencionados anteriormente son indicativos pero no generalizables. Por su parte, la información proporcionada por la encuesta es representativa de la intención, pero no de la acción de trasladarse de EPS. El objetivo de este estudio es cuantificar la magnitud del fenómeno del traslado entre EPS en los últimos catorce años para una cohorte definida de adultos, utili- zando una muestra semialeatoria de historias laborales que permita atenuar el problema de selección. Para esto se hizo uso de la información pública disponible en la base única de afiliación del Fondo de Solidaridad y Garantía (Fosyga), correspondiente a historias de cotización al sistema de seguridad social en salud. De acuerdo con la evidencia anteriormente presentada, la hipótesis de trabajo es que la tasa de traslados es baja. De comprobarse empíricamente, la interpretación apuntaría hacia fallas en el sistema de rendición de cuentas diseñado dentro del sistema que deben ser corregidas.

Tabla 1. Intención de traslado de eps según Encuesta de Evaluación de Servicios de eps 2013

\begin{tabular}{|c|c|c|c|}
\hline Pregunta & Respuestas & Total EPS & $\begin{array}{l}\text { Total EPS } \\
\text { régimen } \\
\text { contributivo }\end{array}$ \\
\hline \multirow{3}{*}{$\begin{array}{l}\text { ¿Ha pensado } \\
\text { en cambiarse a } \\
\text { otra EPS? }\end{array}$} & Sí & $13,3 \%$ & $16,1 \%$ \\
\hline & No & $86,7 \%$ & $83,9 \%$ \\
\hline & Total & $100,0 \%$ & $100,0 \%$ \\
\hline \multirow{15}{*}{$\begin{array}{l}\text { ¿Cuál es el } \\
\text { principal } \\
\text { motivo por el } \\
\text { que ha pensado } \\
\text { en cambiarse } \\
\text { de EPs? }\end{array}$} & $\begin{array}{l}\text { No recibe un trato amable y cordial por parte del } \\
\text { personal de la EPS }\end{array}$ & $3,9 \%$ & $2,9 \%$ \\
\hline & Hay demasiadas demoras para recibir los servicios & $31,3 \%$ & $30,8 \%$ \\
\hline & $\begin{array}{l}\text { No ha tenido respuestas efectivas ante sus problemas } \\
\text { de salud }\end{array}$ & $22,0 \%$ & $22,9 \%$ \\
\hline & $\begin{array}{l}\text { Dificultad de trámites para acceder a los servicios de } \\
\text { salud de la EPS }\end{array}$ & $8,3 \%$ & $8,6 \%$ \\
\hline & $\begin{array}{l}\text { Disminución en la red de prestadores (centros médi- } \\
\text { cos, puntos de atención) }\end{array}$ & $7,0 \%$ & $6,3 \%$ \\
\hline & $\begin{array}{l}\text { Por motivos personales (cambio de domicilio, cambio } \\
\text { de ciudad) }\end{array}$ & $1,1 \%$ & $0,9 \%$ \\
\hline & Cambio en la calidad de la atención ofrecida por la EPS & $11,0 \%$ & $13,7 \%$ \\
\hline & Deficiencias en las instalaciones & $0,8 \%$ & $0,9 \%$ \\
\hline & Sitios de atención muy distantes & $2,5 \%$ & $2,7 \%$ \\
\hline & Otra EPS le ofrece mejores servicios & $7,8 \%$ & $6,2 \%$ \\
\hline & Para unificar el grupo familiar & $1,2 \%$ & $0,8 \%$ \\
\hline & Por recomendación de un familiar o conocido & $1,1 \%$ & $0,8 \%$ \\
\hline & Otro & $2,0 \%$ & $2,6 \%$ \\
\hline & No informa & $0,0 \%$ & $0,0 \%$ \\
\hline & Total & $100,0 \%$ & $100,0 \%$ \\
\hline
\end{tabular}

Fuente: Ministerio de Salud y Protección Social 
Este documento está compuesto por cuatro secciones, incluida esta introducción. En la segunda sección se describe la metodología. En la tercera se presentan los resultados y la última sección discute las implicaciones del estudio.

\section{Metodología}

La fuente de información es la base de datos de compensación publicada por el Fosyga (3). Se denomina Maestro Afiliados Compensados y puede ser consultada introduciendo el tipo de documento (cédula de ciudadanía, tarjeta de identidad, cédula de extranjería, pasaporte, registro civil, Número Único de Identificación Personal). El siguiente texto acompaña la página de consulta y sugiere que esta base de datos es pública, para evitar la negación en la prestación de servicios:

El Ministerio de la Protección Social comunica que la información dispuesta en esta consulta contiene los datos reportados conforme a las fechas definidas en los decretos 2280 de 2005 y 1013 de 1998 por las Empresas Promotoras de Salud - EPS y Entidades Obligadas a Compensar - EOC que han superado el proceso de validación y cruce definidos en las normas y en las especificaciones técnicas; por lo tanto esta información se debe utilizar como complemento al marco legal y técnico definido y nunca como único criterio para denegar la prestación de los servicios de salud a las personas. Si usted encuentra una inconsistencia en la información publicada, por favor remítase a la EPS o EOC y solicite la corrección de su información a fin de que esta remita la novedad correspondiente al Consorcio SAYP 2011 y esta proceda a la actualización en las bases de datos.

En las tablas 2 y 3 se presenta la estructura de la base de datos proporcionada en el portal de Fosyga. Para cada cédula de ciudadanía (CC) se encuentran disponibles nombres y apellidos, EPs a la que cotizó, el periodo compensado (mes, año), la fecha de afiliación (día, mes, año), el número de días compensados (0-30), el tipo de afiliado (cotizante/ beneficiario) y la norma que aplica en el momento de compensación (2309/2280/4023).

Para este estudio se extrajo de la base de datos la historia de afiliación de hombres y mujeres nacidos entre 1972 y 1973 . El procedimiento tuvo dos pasos: 1) se identificaron diez cédulas de personas conocidas por el autor y se definieron como cédula semilla; 2) se extrajeron

TABLA 2. INFORMACIÓN BÁSICA DEL AFILIADo

\begin{tabular}{|c|c|c|c|c|c|c|c|c|}
\hline $\begin{array}{c}\text { Tipo } \\
\text { ID }\end{array}$ & $\begin{array}{c}\text { Número de } \\
\text { documento }\end{array}$ & $\begin{array}{c}\text { Primer } \\
\text { apellido }\end{array}$ & $\begin{array}{c}\text { Segundo } \\
\text { apellido }\end{array}$ & $\begin{array}{c}\text { Primer } \\
\text { nombre }\end{array}$ & $\begin{array}{c}\text { Segundo } \\
\text { nombre }\end{array}$ & $\begin{array}{c}\text { Último } \\
\text { periodo } \\
\text { compensado }\end{array}$ & $\begin{array}{c}\text { EPS / } \\
\text { EOC }\end{array}$ & $\begin{array}{c}\text { Tipo } \\
\text { afiliación }\end{array}$ \\
\hline CC & XXXXXX & Prada & Ríos & Sergio & Iván & MM/YYYY & EPS. XX & $\begin{array}{c}\text { Cotizante - } \\
\text { dependiente }\end{array}$ \\
\hline
\end{tabular}

Fuente: Fosyga

TABLA 3. INFORMACIÓN DE PERIODOS COMPENSADOS

\begin{tabular}{|l|l|l|l|l|l|}
\hline EPS / EOC & $\begin{array}{l}\text { Periodos } \\
\text { compensados }\end{array}$ & $\begin{array}{l}\text { Fecha de } \\
\text { afiliación }\end{array}$ & $\begin{array}{l}\text { Días } \\
\text { compensados }\end{array}$ & Tipo afiliado & Norma \\
\hline EPS N & $03 / 2014$ & DD/MM/YYYY & 30 & Cotizante & 4023 \\
\hline EPS N & $02 / 2014$ & DD/MM/YYYY & 30 & Cotizante & 4023 \\
\hline EPS N & $01 / 2014$ & DD/MM/YYYY & 30 & Cotizante & 4023 \\
\hline
\end{tabular}

Fuente: Fosyga 
cien historias laborales existentes hacia adelante y cien hacia atrás, agregando o restando una unidad al número de identificación. Así, por ejemplo, si la cc termina en el número 345 , hacia adelante se busca la terminada en 346, y hacia atrás la terminada en 344. Sí la historia existe se ingresa a la base de datos, si no se continúa hasta encontrar la siguiente.

Inicialmente, la base de datos contiene 1034 cédulas únicas, con 115698 observaciones. Se realiza una correspondencia entre el sexo y la ciudad de expedición de la cédula con el registro de compensación de cada cédula. La asignación del número serial de la cc solo tiene que ver con el género y la ciudad (por ejemplo, las cedulas 24.Xxx.xxx son para mujeres en Manizales, mientras que las 7x.xxx. xxx son para hombres en Medellín) y el orden de llegada a la Registraduría. De tal forma que la selección de la muestra puede considerarse semialeatoria, dado que el número semilla no es aleatorio, pero no hay correlación entre CC consecutivas. La distribución por género y ciudad se presenta en la tabla 4.

\section{Criterios de inclusión}

Se eliminan observaciones anteriores a enero del 2001, debido a que la información de la base de datos no es confiable. Como resultado se obtienen 1028 cédulas únicas y 114528 observaciones. De este conjunto de CC se encuentran individuos con pocos meses de compensación. Por esta razón, se define otro criterio de inclusión: solo individuos que presentan doce o más periodos de cotización ( este criterio se eliminan 358 periodos cotizados y permanecen 950 individuos. El 87,7\% de los periodos cotizados tiene treinta días cotizados a una EPS.

Los cotizantes pueden dividirse en tres grupos: 1) personas que siempre fueron cotizantes (no necesariamente de manera ininterrumpida), 2) personas que fueron cotizantes y beneficiarios, y 3 ) aquellos que solo fueron beneficiarios. El tercer criterio de inclusión es ser solo cotizante. En términos prácticos, esta distinción es importante para el estudio porque es el cotizante quien toma la decisión de trasladarse o no de EPS. La distribución de los 950 individuos por ciudad y por tipo de afiliación se presenta en la tabla 5. Solo cotizantes son 404 , de los cuales el $37,4 \%$ son mujeres y un $62,6 \%$ son hombres; afiliados en algún momento a 25 EPs distintas. Como resultado de estas exclusiones, el 91,75\% de las observaciones tiene 30 días de cotización.

\section{Depuración de los datos}

En el proceso de depuración se borraron los registros de cero días compensados (2286 observaciones). Se encontraron duplicados de dos tipos en un mismo mes: 1) por diferencias en la fecha de afiliación, 2) por

TABLA 4. DistribuCión POR GÉNERo Y CIUdAd de LA MUESTRA INICIAL

\begin{tabular}{|l|l|l|l|}
\hline Ciudad & Mujeres & Hombres & Total \\
\hline Bogotá & 101 & 103 & 204 \\
\hline Bucaramanga & 112 & 106 & 218 \\
\hline Cali & 100 & 106 & 206 \\
\hline Manizales & 106 & 100 & 206 \\
\hline Medellín & 100 & 100 & 200 \\
\hline Total & 519 & 515 & 1034 \\
\hline
\end{tabular}

Fuente: Fosyga - cálculos propios 
TABla 5. DistribuCión POR CIUdAd, SEXo Y TIPO DE AFILIACIÓN

\begin{tabular}{|l|l|l|l|}
\hline Ciudad & Mujeres & Hombres & Total \\
\hline Bogotá & 99 & 95 & 194 \\
\hline Bucaramanga & 107 & 95 & 202 \\
\hline Cali & 94 & 92 & 186 \\
\hline Manizales & 94 & 89 & 183 \\
\hline Medellín & 96 & 89 & 185 \\
\hline Tipo de afiliación & \multicolumn{2}{|l|}{} \\
\hline Siempre cotizante & 151 & 253 & 404 \\
\hline Beneficiario/cotizante & 238 & 195 & 433 \\
\hline Siempre beneficiario & 101 & 12 & 113 \\
\hline Total & 490 & 460 & 950 \\
\hline
\end{tabular}

Fuente: Fosyga - cálculos propios

fraccionamiento en los días de cotización. Estos problemas se corrigieron sumando los días de cotización por cédula, año y mes, sin tener en cuenta la fecha de afiliación. Adicionalmente, se eliminaron 41 observaciones exactamente iguales y los duplicados generados por la cotización simultánea a dos EPS. Se presentaron casos atípicos en los que la suma de los días excedió los treinta días, por lo que se optó por dejar el que tuviera el mayor número de días cotizados. La distribución de periodos según EPs se presenta en la tabla 6 , y la distribución por cédulas (por afiliados) para el año 2014 en la tabla 7. En esta última tabla se puede comparar la muestra con el valor poblacional, y se encuentran diferencias importantes en Saludcoop y Sura, lo cual es explicado por la ubicación geográfica y la edad de la cohorte.

\section{Análisis de resultados}

La base de datos construida permite responder múltiples preguntas, de forma tal que esta sección sigue un formato de preguntas y respuestas.
¿En promedio cuántos meses cotizó un individuo de esta cohorte en los últimos catorce años?

La figura 1 muestra la distribución de meses cotizados y la tabla 8 la estadística descriptiva. En promedio una persona cotizó 115 meses (9,6 años), pero de acuerdo con la mediana la mitad de los individuos cotizaron 135 meses (11,25 años) o más. Un cuarto de las personas cotizó 162 periodos o más (13,5 años), y aproximadamente el $10 \%$ de las personas en la muestra cotizó de manera ininterrumpida los catorce años del estudio. Como se puede observar, la mayoría de individuos tuvieron una cotización fragmentada, con momentos en los cuales no registraron periodos compensados.

¿Cuántos periodos de cotización continuos tiene una persona?

Se caracteriza cómo periodo continuo a los meses consecutivos en los cuales la persona cotizó en el sistema de salud. En la tabla 9 se presentan la distribución de las 404 cédulas según la cantidad de periodos cotizados, el mínimo y máximo de meses por periodo. Dentro del grupo de cotización continua, 
correspondiente a 168 meses, se clasifico el $6,4 \%$ de las personas. Asimismo, el 6,7\% de los individuos tuvo una cotización continua, pero menor al número total, con una media de 6,6 años. Adicionalmente, porcentajes similares de personas tuvieron dos y tres periodos continuos de cotización respectivamente $(15 \%)$. Resalta que más de la mitad de los individuos en la muestra presentó tres o más periodos de cotización.

\section{¿Dentro de un mismo periodo continuo de cotización cuántos traslados hay?}

En el cálculo del indicador de traslados se excluyeron veintiséis cambios en el periodo de estudio, debido a que la fecha en la que se efectuaron coincidía con el proceso de liquidación de la entidad prestadora de salud de la cual estaban saliendo. Así, estos cambios

Tabla 6. Periodos cotizados por eps Para 404 historias laborales entre 2001-2014

\begin{tabular}{|c|c|c|c|}
\hline EPS & $\begin{array}{l}\text { Periodos } \\
\text { cotizados }\end{array}$ & $\%$ & $\%$ acumulado \\
\hline Coomeva & 6923 & 14,92 & 14,92 \\
\hline SURA & 5546 & 11,95 & 26,88 \\
\hline Nueva EPs (Instituto de Seguros Sociales) & 4844 & 10,44 & 37,32 \\
\hline Salud Total S.A. & 4692 & 10,11 & 47,43 \\
\hline Saludcoop & 4482 & 9,66 & 57,09 \\
\hline Servicio Occidental de Salud & 3151 & 6,79 & 63,89 \\
\hline Sanitas S.A. & 3147 & 6,78 & 70,67 \\
\hline Comfenalco Valle & 1964 & 4,23 & 74,90 \\
\hline Cruz Blanca & 1935 & 4,17 & 79,07 \\
\hline Comfenalco Antioquia & 1758 & 3,79 & 82,86 \\
\hline Famisanar Ltda. & 1623 & 3,50 & 86,36 \\
\hline Compensar & 1520 & 3,28 & 89,64 \\
\hline Solsalud S.A & 1111 & 2,39 & 92,03 \\
\hline Aliansalud & 1076 & 2,32 & 94,35 \\
\hline Cafesalud S.A. & 1075 & 2,32 & 96,67 \\
\hline Colpatria & 850 & 1,83 & 98,5 \\
\hline Humana Vivir S.A. & 219 & 0,47 & 98,97 \\
\hline Saludvida S.A. & 137 & 0,30 & 99,27 \\
\hline Cajanal EPs & 84 & 0,18 & 99,45 \\
\hline Red Salud Atención Humana & 58 & 0,13 & 99,58 \\
\hline Colseguros & 52 & 0,11 & 99,69 \\
\hline EPS de Caldas & 52 & 0,11 & 99,8 \\
\hline SALUDCOLOMBIA & 51 & 0,11 & 99,91 \\
\hline Unimec & 40 & 0,09 & 100 \\
\hline Multimédicas Salud con Calidad S.A. & 2 & 0 & 100 \\
\hline Total & 46392 & 100 & \\
\hline
\end{tabular}

Fuente: Fosyga - cálculos propio 
Tabla 7. DistribuCión de AFILIAdos EN MUESTRA Y EN POBLACIÓN PARA 2014

\begin{tabular}{|l|c|c|}
\hline Nombre EPS & Muestra & Población* \\
\hline SURA & $18,0 \%$ & $10.0 \%$ \\
\hline Coomeva & $13,8 \%$ & $14,6 \%$ \\
\hline Nueva EPS (Instituto de Seguros Sociales) & $10,7 \%$ & $12,3 \%$ \\
\hline Saludcoop & $10,4 \%$ & $19,6 \%$ \\
\hline Salud Total S.A & $9,7 \%$ & $9,5 \%$ \\
\hline Sanitas S.A. & $9,3 \%$ & $6,6 \%$ \\
\hline Servicio Occidental de Salud & $8,3 \%$ & $4,5 \%$ \\
\hline Famisanar Ltda. & $5,5 \%$ & $7,9 \%$ \\
\hline Comfenalco Valle & $4,2 \%$ & $1,7 \%$ \\
\hline Compensar & $3,8 \%$ & $5,2 \%$ \\
\hline Cruz Blanca & $2,4 \%$ & $2,8 \%$ \\
\hline Aliansalud & $2,1 \%$ & $1,4 \%$ \\
\hline Cafesalud & $1,4 \%$ & $3,6 \%$ \\
\hline Saludvida & $0,3 \%$ & $0,4 \%$ \\
\hline
\end{tabular}

Fuente: Fosyga - cálculos propios

*Valor poblacional tomado de la BDUA, para el total de personas entre 40 y 50 años afiliadas a salud por tipo de régimen según EPS con fecha de corte marzo del 2015.

TABla 8. Estadística descriptiva: nÚMERo de MESES COTIZAdos POR INDIVIDUO

\begin{tabular}{|l|l|}
\hline Percentil & Meses \\
\hline 1 & 12 \\
\hline 5 & 21 \\
\hline 10 & 30 \\
\hline 25 & 69,5 \\
\hline 50 & 135 \\
\hline 75 & 162 \\
\hline 90 & 167 \\
\hline 95 & 168 \\
\hline 99 & 168 \\
\hline Media & 115 \\
\hline Moda & 168 \\
\hline Desv St & 51,7 \\
\hline
\end{tabular}

Fuente: Fosyga - cálculos propios 
Figura 1. Distribución de MESES cotizados

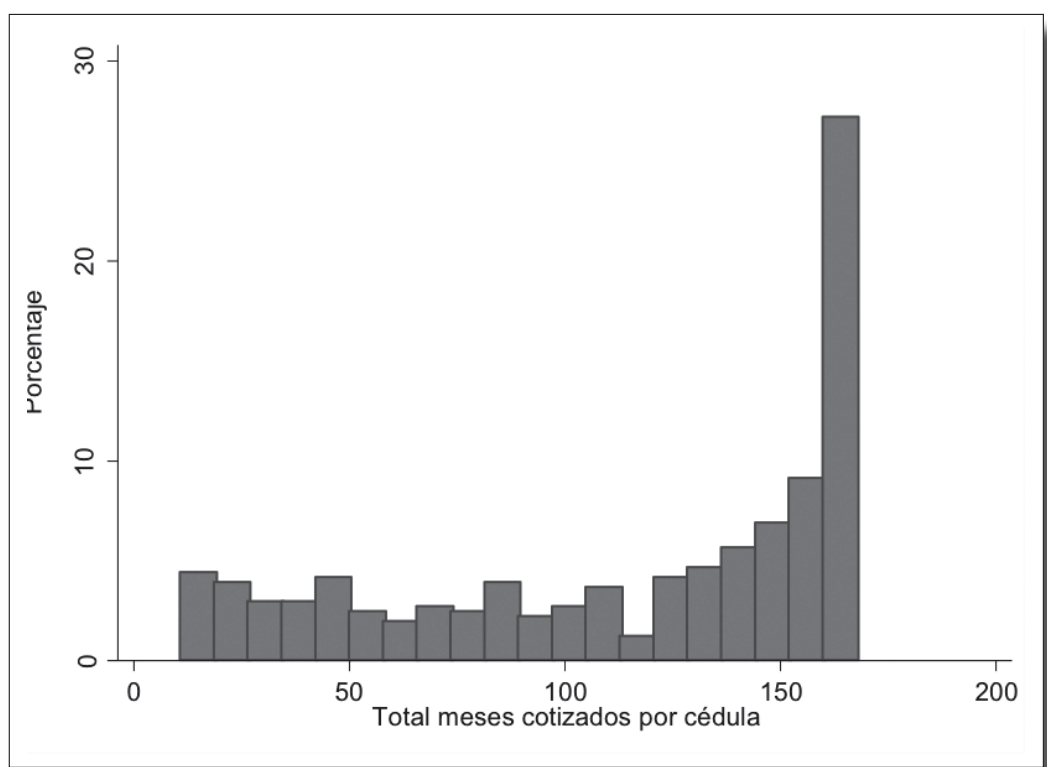

Fuente: Fosyga - cálculos propios

TABla 9. NúMERo de Periodos CONTINUOS DE COTIZACIÓN

\begin{tabular}{|l|c|c|c|c|}
\hline Categoría & Obs. & $\%$ & Mín. & Máx. \\
\hline Cotización continua & 26 & $6,4 \%$ & 168 & 168 \\
\hline Cotiza 1 periodo & 27 & $6,7 \%$ & 12 & 167 \\
\hline Cotiza 2 periodos & 61 & $15,1 \%$ & 11 & 167 \\
\hline Cotiza 3 periodos & 62 & $15,3 \%$ & 11 & 167 \\
\hline Cotiza 4 periodos & 61 & $15,1 \%$ & 13 & 167 \\
\hline Cotiza 5 periodos & 41 & $10,1 \%$ & 12 & 167 \\
\hline Cotiza 6 periodos & 32 & $7,9 \%$ & 33 & 166 \\
\hline Cotiza 7 periodos & 26 & $6,4 \%$ & 39 & 162 \\
\hline Cotiza 8 periodos & 12 & $3,0 \%$ & 21 & 162 \\
\hline Cotiza 9 periodos & 15 & $3,7 \%$ & 17 & 158 \\
\hline Cotiza 10 periodos & 13 & $3,2 \%$ & 18 & 158 \\
\hline Cotiza 11 periodos o más & 28 & $6,9 \%$ & 18 & 153 \\
\hline
\end{tabular}

Fuente: Fosyga - cálculos propios

se toman como obligatorios y no reflejan la decisión propia del usuario.

La tabla 10 muestra el número de personas que hacen traslados de EPS y el número de traslados totales dentro del periodo continuo.
Se debe tener en cuenta que estos periodos no distinguen entre años de cotización. La categoría correspondiente a los usuarios que cotizan durante cuatro periodos o más presenta tanto el mayor número de personas que se cambian como el mayor número de cambios totales (73 y $79 \%$, respectivamente). Quienes cotizaron 
TABla 10. IndiCAdores de traslados POR PERIOdOS CONTINUO DE COTIZACIÓN

\begin{tabular}{|c|c|c|c|c|c|c|c|}
\hline $\begin{array}{l}\text { Categoría por número } \\
\text { de periodos cotizados }\end{array}$ & Total & $\begin{array}{l}\text { Personas } \\
\text { que } \\
\text { realizan } \\
\text { traslado }\end{array}$ & $\begin{array}{l}\text { Personas } \\
\text { que no } \\
\text { realizan } \\
\text { traslado }\end{array}$ & $\begin{array}{l}\text { Número } \\
\text { de } \\
\text { traslados }\end{array}$ & $\begin{array}{c}\text { Porcentaje } \\
\text { de personas } \\
\text { que no se } \\
\text { trasladan }\end{array}$ & $\begin{array}{l}\text { Número } \\
\text { de } \\
\text { traslados } \\
\text { por } \\
\text { afiliado }\end{array}$ & $\begin{array}{c}\text { Número } \\
\text { de } \\
\text { traslados } \\
\text { por } \\
\text { afiliados } \\
\text { que se } \\
\text { traslada }\end{array}$ \\
\hline Cotización continua & 26 & 10 & 16 & 12 & $61,5 \%$ & 0,462 & 1,20 \\
\hline 1 periodo & 27 & 0 & 27 & 0 & $100,0 \%$ & - & - \\
\hline 2 periodos & 61 & 4 & 57 & 4 & $93,4 \%$ & 0,066 & 1,00 \\
\hline 3 periodos & 62 & 14 & 48 & 14 & $77,4 \%$ & 0,226 & 1,00 \\
\hline 4 periodos & 61 & 20 & 41 & 21 & $67,2 \%$ & 0,344 & 1,05 \\
\hline 5 periodos & 41 & 15 & 26 & 18 & $63,4 \%$ & 0,439 & 1,20 \\
\hline 6 periodos & 32 & 8 & 24 & 15 & $75,0 \%$ & 0,469 & 1,88 \\
\hline 7 periodos & 26 & 6 & 20 & 13 & $76,9 \%$ & 0,500 & 2,17 \\
\hline 8 periodos & 12 & 2 & 10 & 3 & $83,3 \%$ & 0,250 & 1,50 \\
\hline 9 periodos & 15 & 5 & 10 & 7 & $66,7 \%$ & 0,467 & 1,40 \\
\hline 10 periodos & 13 & 6 & 7 & 6 & $53,8 \%$ & 0,462 & 1,00 \\
\hline 11 periodos o más & 28 & 12 & 16 & 27 & $57,1 \%$ & 0,964 & 2,25 \\
\hline
\end{tabular}

Fuente: Fosyga - cálculos propios

Tabla 11. Traslados de eps POR año Y Meses de cotización

\begin{tabular}{|c|c|c|c|c|}
\hline & \multicolumn{2}{|c|}{ Cotizan doce meses } & \multicolumn{2}{c|}{ Cotizan entre cuatro y once meses } \\
\hline Año & $\mathbf{N}$ & $\mathbf{T}$ & 124 & $\mathbf{T}$ \\
\hline 2001 & 137 & $5,84 \%$ & 126 & $4,03 \%$ \\
\hline 2002 & 150 & $2,67 \%$ & 113 & $3,97 \%$ \\
\hline 2003 & 175 & $3,43 \%$ & 101 & $2,65 \%$ \\
\hline 2004 & 193 & $2,07 \%$ & 109 & $3,96 \%$ \\
\hline 2005 & 199 & $3,52 \%$ & 76 & $0,92 \%$ \\
\hline 2006 & 222 & $3,15 \%$ & 90 & $3,33 \%$ \\
\hline 2007 & 218 & $4,13 \%$ & 79 & $2,53 \%$ \\
\hline 2008 & 224 & $0,89 \%$ & 66 & $6,06 \%$ \\
\hline 2009 & 231 & $2,16 \%$ & 68 & $5,88 \%$ \\
\hline 2010 & 229 & $3,06 \%$ & 63 & $4,76 \%$ \\
\hline 2011 & 241 & $3,73 \%$ & 51 & $3,92 \%$ \\
\hline 2012 & 250 & $1,60 \%$ & 59 & $5,08 \%$ \\
\hline 2013 & 245 & $3,67 \%$ & 50 & $8,00 \%$ \\
\hline 2014 & 253 & $2,37 \%$ & & $00 \%$ \\
\hline
\end{tabular}

$\mathrm{N}=$ tamaño de muestra, $\mathrm{T}=$ personas que se mueven 
solo un periodo continuo, no presentaron cambios a lo largo de los años. En el caso de las categorías de cotización continua y cotización de cuatro periodos o más, se evidencia que algunas personas efectuaron el traslado a otra EPS más de una vez, siendo el número de cambios mayor al número de cédulas. Las categorías correspondientes a la cotización de dos y tres periodos presentaron el mismo número de personas como de cambios totales.

\section{¿En un año calendario qué porcentaje de} personas se trasladan de EPS?

Para responder a esta pregunta se divide la muestra en tres grupos: aquellos que tienen cotización los doce meses del año, aquellos que tienen más de cuatro y menos de doce meses, y aquellos que tienen tres periodos o menos. Los resultados para las dos primeras poblaciones se presentan en la tabla 11. En el grupo de cotizantes que estuvo compensado los doce meses del año se encuentra que la proporción de personas que efectuó un traslado de EPS dentro del primer grupo de cotizantes no supera el $6 \%$ en ningún año, y asimismo, que la proporción de personas que cumple con este criterio es más del $50 \%$ y está en una tendencia creciente. En el grupo que cotiza entre cuatro y once meses, las tasas de traslado también son bajas y oscilan entre 1 y $8 \%$, pero sobresale el hecho de que la proporción está en descenso.

¿En periodos de más años qué porcentaje de personas se trasladan de EPS?

La tabla 12 muestra el mismo cálculo anterior para periodos más extensos. Se caracterizan ventanas para dos, tres, cuatro y cinco años consecutivos. Debido a que el número de periodos aumenta, el análisis se concentra en todos los cotizantes de la

TABla 12. TASa de traslados POR PERíodos ANUALES

\begin{tabular}{|c|c|c|c|c|c|}
\hline & \multirow[b]{2}{*}{ Periodo } & \multicolumn{2}{|c|}{ Muestra } & \multicolumn{2}{|c|}{ Cotizan todos los meses } \\
\hline & & $\mathbf{N}$ & M & $\mathbf{N}$ & M \\
\hline \multirow{7}{*}{ Dos años } & 2001-02 & 404 & $5,20 \%$ & 91 & $5,49 \%$ \\
\hline & 2003-04 & 404 & $4,21 \%$ & 142 & $7,04 \%$ \\
\hline & 2005-06 & 404 & $3,71 \%$ & 170 & $7,65 \%$ \\
\hline & 2007-08 & 404 & $3,96 \%$ & 183 & $4,92 \%$ \\
\hline & $2009-10$ & 404 & $5,20 \%$ & 203 & $5,42 \%$ \\
\hline & 2011-12 & 404 & $4,46 \%$ & 218 & $5,50 \%$ \\
\hline & 2013-14 & 404 & $5,20 \%$ & 224 & $5,80 \%$ \\
\hline \multirow{4}{*}{ Tres años } & 2003-05 & 404 & $5,94 \%$ & 109 & $11,01 \%$ \\
\hline & 2006-08 & 404 & $5,69 \%$ & 163 & $7,36 \%$ \\
\hline & 2009-11 & 404 & $7,43 \%$ & 189 & $7,94 \%$ \\
\hline & $2012-14$ & 404 & $6,44 \%$ & 208 & $7,21 \%$ \\
\hline \multirow{3}{*}{ Cuatro años } & 2003-06 & 404 & $7,67 \%$ & 93 & $16,13 \%$ \\
\hline & $2007-10$ & 404 & $8,91 \%$ & 151 & $9,93 \%$ \\
\hline & 2011-14 & 404 & $9,16 \%$ & 188 & $11,70 \%$ \\
\hline \multirow{2}{*}{ Cinco años } & 2005-09 & 404 & $9,90 \%$ & 121 & $10,74 \%$ \\
\hline & $2010-14$ & 404 & $11,63 \%$ & 168 & $14,29 \%$ \\
\hline
\end{tabular}

$\mathrm{N}=$ tamaño de muestra, $\mathrm{T}=$ personas que se mueven

Fuente: Fosyga - cálculos propios 
Figura 2. Cambios realizados hacia una EPS, PERIOdo 2001-2014

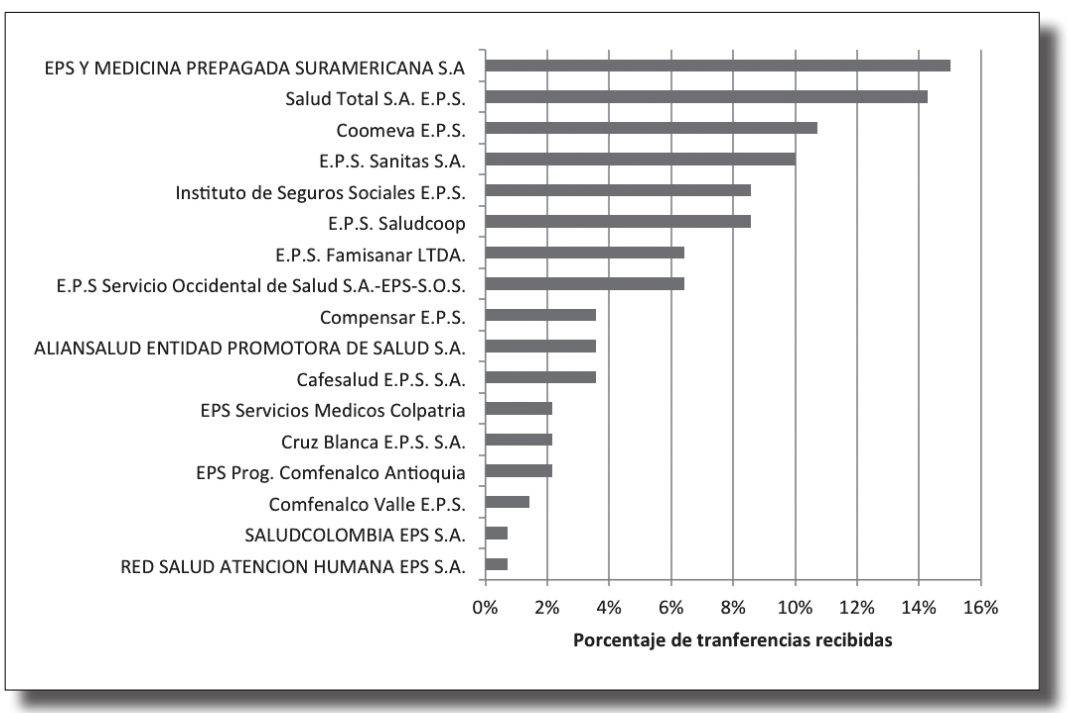

Fuente: Fosyga - cálculos propios

Figura 3. Retiros efectuados POR EPS, PERIOdo 2001-2014

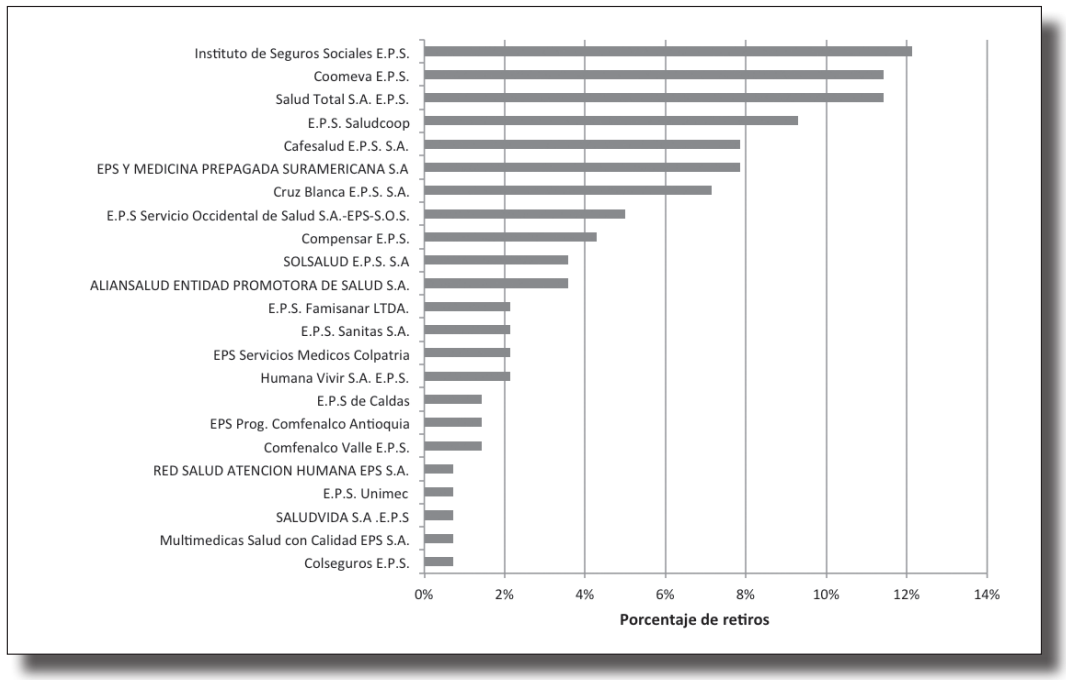

Fuente: Fosyga - cálculos propios

cohorte y en la submuestra de los que están compensados todos los meses del periodo en cuestión. Como es de esperar, con la ampliación del número de meses aumenta la probabilidad de traslados, no obstante, aun 188 de traslado se da en el último quinquenio, con uno de cada siete cotizantes efectuando un traslado de EPs. Para la cohorte total, las tasas también aumentan con el número de años, siendo la tasa más alta la observada para el periodo 2010-2014 con un 11\% de personas trasladándose de EPS, es decir, una de cada nueve personas. 
¿Qué revelan las preferencias de los que se trasladan?

La base de datos permite identificar las EPS que reciben la mayor proporción de cambios y aquellas de las cuales los usuarios tienden a retirarse durante el periodo de estudio. En la figura 3 se ordenan, de mayor a menor, las EPS a las que más personas se trasladaron. La EPS que obtuvo el mayor número de ingresos fue EPS Sura, seguida por Salud Total, Coomeva y EPS Sanitas.

La figura 3 muestra el porcentaje de retiros por EPS, evidenciando que La Nueva EPS (antiguo Instituto de Seguros Sociales) es la que más deserción tiene, mientras que Coomeva y Salud Total ocupan el segundo puesto. Resalta el hecho de que Salud Total ocupe el segundo puesto, tanto en ingresos como en retiros realizados por los usuarios, dando la idea de que esta EPS tiene una rotación de usuarios más alta en comparación con las demás.

¿Existe alguna relación entre las preferencias reveladas e indicadores de calidad?

La respuesta es: parcialmente. En la tabla 13 se presenta el ordenamiento establecido según la Encuesta de Evaluación de los Servicios de las EPs (4). Esta clasificación incorpora la percepción de los usuarios, por medio de catorce preguntas realizadas dentro de la encuesta (en torno a la calidad del servicio, tiempos de espera y facilidad en los trámites). Como puede observarse, sí existe alguna correlación. Por ejemplo, la

Tabla 13. Encuesta de Evaluación de Servicios de las eps

\begin{tabular}{|l|l|}
\hline \multirow{4}{*}{ Grupo } & EPS \\
\hline \multirow{5}{*}{ Medio Alto } & AlianSalud \\
\cline { 2 - 3 } & Comfenalco Valle EPS \\
\hline & EPS Programam Comfenalco Antioquia \\
\cline { 2 - 3 } & EPS SURA \\
\hline & Cafe Salud \\
\cline { 2 - 3 } & Colpatria \\
\hline & Compensar \\
\hline \multirow{5}{*}{ Medio Bajo } & Cruz blanca \\
\hline & Salud total \\
\hline & Sanitas \\
\hline & Coomeva \\
\hline & Nueva EPS \\
\hline & Saludcoop \\
\hline & EPS s.o.s \\
\hline \multirow{5}{*}{ Bajo } & Farmisanar \\
\hline & Golden Group \\
\hline & Humana Vivir \\
\hline & Salud Vida \\
\hline & Sol Salud \\
\hline
\end{tabular}

Fuente: Minsalud Encuesta de Evaluación de los Servicios de las EPs 2012 
EPS Sura cuenta con el mayor porcentaje de traslados hacia una EPS y pertenece al nivel alto del ranking. La Nueva EPS, que reporta la mayor cantidad de retiros, se encuentra en el nivel medio-bajo del ranking.

No obstante, al analizar cuatro dimensiones específicas evaluadas dentro de la encuesta (tiempo de asignación de citas de medicina general, tiempo en la asignación de citas con especialistas, tiempo de entrega de autorizaciones y la entrega total de medicamentos), con respecto a los resultados obtenidos, se verifica la inexistencia de una relación clara.

En la figura 4 se aprecia la correlación entre el porcentaje de cambios recibidos y el porcentaje de usuarios que opinan que el servicio en cada una de las dimensiones es óptimo. Así, inicialmente se observa la relación entre el porcentaje de cambios recibidos por cada EPS y el porcentaje de usuarios que opinan que el tiempo de asignación de una cita de medicina general oscila entre cero y tres días. Lo mismo sucede para el tiempo de asignación de citas con el especialista y el tiempo de entrega de autorizaciones. En el caso de los medicamentos, se relacionan los cambios hacia la EPS con el porcentaje de usuarios que afirman que les entregaron todos los medicamentos recetados por el médico.

En efecto, no existe una correlación clara entre el porcentaje de cambios recibidos por una EPS y la opinión de los usuarios en torno a estas dimensiones. Por ejemplo, se presentan EPS con respecto a las cuales los usuarios opinan que la respuesta a la solicitud de citas es óptima, pero aun así no captaron cambios durante el periodo. Hay otras que teniendo un porcentaje alto de opiniones favorables, efectivamente captan un mayor porcentaje de cambios. Finalmente, existen EPS que teniendo un porcentaje más bajo en torno a las opiniones de los usuarios, tienen un numero de cambios alto. Por tanto, no hay evidencia para establecer que las personas tienden

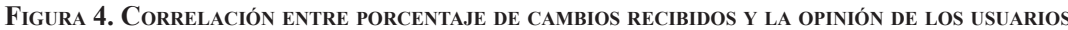

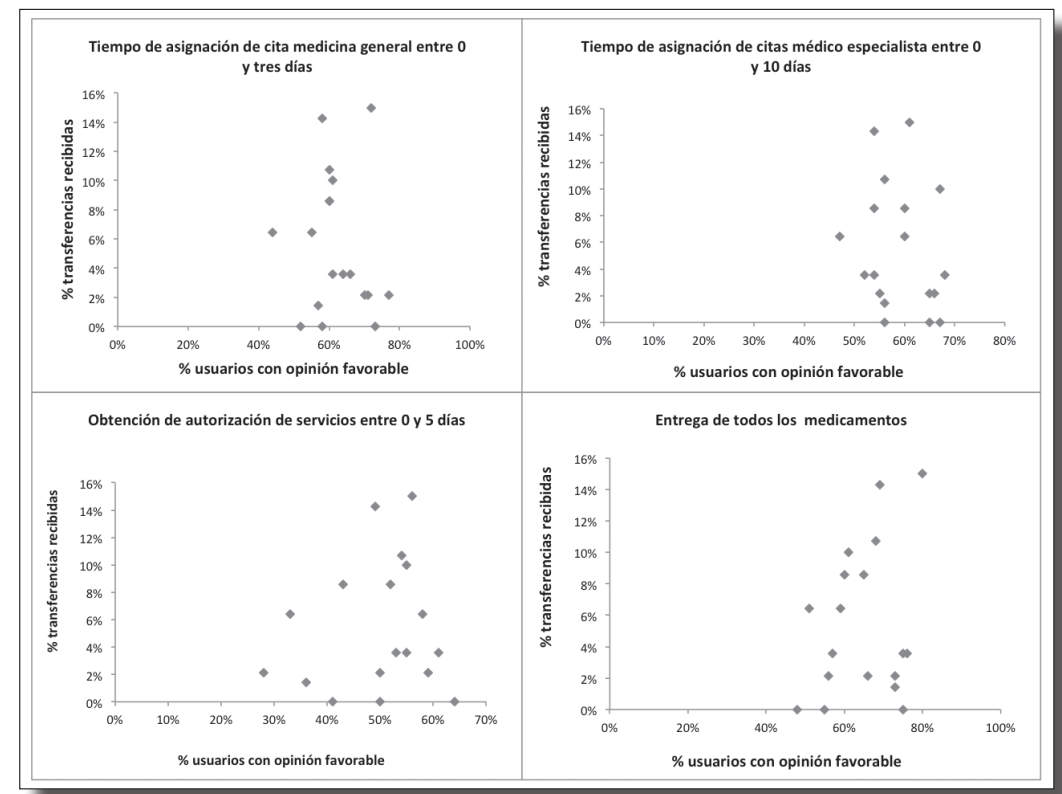

Fuente: Fosyga, Minsalud - cálculos propios 
a cambiarse a las EPS que reportan niveles altos en la calidad de los servicios prestados.

\section{Discusión}

Después de veinte años de entrada en vigencia, no existe en Colombia un estudio sistemático de las dinámicas de traslados entre EPS. Estudiar esta dinámica es importante porque uno de los pilares fundamentales del modelo de competencia regulada es la libre escogencia de EPS como mecanismo de rendición de cuentas. Existen razones para pensar que esto no es de interés para las EPS y/o sus agremiaciones. Las de mejor calidad, por el temor de que los individuos más enfermos decidan irse a estas (5), y las de calidad inferior, por el temor a desaparecer del mercado. Además del poco interés de las EPS, existen restricciones a la información en la fuente (Fosyga), debido a su carácter confidencial.

Este documento presenta la primera aproximación a este cálculo, con base en una novedosa metodología: la escogencia de una cohorte de edad (40 a 45 años) en cinco ciudades principales del país (Bogotá, Medellín, Cali, Bucaramanga y Manizales), de manera semialeatoria, y el seguimiento a las historias laborales de estas personas durante catorce años. Los principales hallazgos del estudio para la cohorte son los siguientes:

- Más del 56\% de las personas tienen cuatro o más periodos de cotización, es decir, que presentan múltiples periodos de inactividad en el sistema.

- Dentro de un mismo periodo de cotización, el porcentaje de las personas que no se cambia de EPS es el mayor.

- En un año calendario, de los que cotizan los doce meses, menos del $6 \%$ decide
- $\quad$ trasladarse, y dentro de los que cotizan menos de doce meses en ese año, menos del $8 \%$ lo hace.

- Incluso en periodos anuales más largos, un quinquenio por ejemplo, la tasa sigue siendo baja, con uno de cada siete cotizantes efectuando un traslado de EPS.

- Se verifica una correlación débil entre traslados e indicadores de calidad de las EPS.

En general, los resultados muestran que a pesar de una alta fragmentación en los periodos de cotización, la tasa de traslados de EPS es baja. La alta fragmentación se explica por las condiciones mismas de la cohorte, tales como altas tasas de desempleo en jóvenes y adultos jóvenes, e interrupciones para estudiar, paternidad o maternidad, etc. Las bajas tasas de traslado entre EPS pueden también ser explicadas por diferentes razones: bajo uso de servicios por ser una cohorte joven, asimetrías en información respecto a la calidad, altos costos de transacción e inercia. De acuerdo con la teoría de la economía del comportamiento humano, una vez que una persona ha hecho una elección o dicha elección ha sido hecha por ella (default), es posible esperar que un gran porcentaje de las personas nunca tomen acciones concretas para cambiarla, incluso cuando la elección no es conveniente para ellas (6-8). Cuál o en qué medida cada uno de estos aspectos explica las bajas tasas de traslado, amerita mayor investigación.

Las limitaciones del estudio son varias. En primer lugar, los hallazgos no son generalizables a otras cohortes. Usuarios más frecuentes del sistema, como los adultos mayores o los enfermos crónicos, darán mayor peso a la continuidad del tratamiento, y por ende tendrán costos de búsqueda más altos. En segundo lugar, los hallazgos no son 
generalizables a zonas dispersas o municipios con baja oferta de prestadores, donde la escogencia no existe.

El hecho de que la tasa de traslados entre EPS sea muy baja, por cualquiera de las razones expuestas y otras que el lector tenga en consideración, sugiere la necesidad de revisar el diseño del mecanismo de rendición de cuentas de los ciudadanos ante las EPs del sistema de salud colombiano.

\section{Referencias bibliográficas}

1. World Bank. World Development Report: Making Services work for Poor People [serie en Internet]. 2004 [acceso: 5 de agosto del 2014]; [aprox. 200 p]. Disponible en: https://openknowledge.worldbank. org/handle/10986/5986.

2. Ministerio de Salud y Protección Social. Encuesta de Evaluación de los Servicios de las EPS [serie en Internet]. 2013 Nov [acceso: 13 de abril del 2015]; [aprox. 1 p.]. Disponible en: http://calidadensalud. minsalud.gov.co/IndicadoresdeCalidad/Resultados/ EncuestadeEPS.aspx.

3. Fosyga [Internet]. Colombia: Fondo de solidaridad y garantía. [fecha desconocida] - [último acceso: $1^{\circ}$ de marzo del 2015]. Disponible en: http://www.fosyga.gov.co/Consultas/AfiliadosCompensados/MaestroAfiliadosCompensados/tabid/439/Default.aspx.

4. Ministerio de Salud y Protección Social. Encuesta de Evaluación de los Servicios de las EPS [serie en Internet]. 2012 Nov [acceso: 13 de abril del 2015]; [aprox. 1 p.]. Disponible en: http://www.supersalud.gov.co/ supersalud/LinkClick.aspx?fileticket $=$ vQFzr $\% 2 \mathrm{Bt}$ gUCM\%3D\&tabid $=58 \&$ mid $=1290$.

5. Castaño R, Zambrano A. Biased selection within the social health insurance market in Colombia. Health Policy. 2006; 79, 313-24.

6. Thaler RH, Sunstein CR. Libertarian paternalism. American Economic Review. 2003 Mayo; 93 (2): $175-9$.

7. Thaler RH, Sunstein CR. Nudge: Improving decisions about health, wealth, and happiness. New Haven, CT: Yale University Press; 2008.

8. World Bank. World Development Report: Mind, Society and Behavior. [serie en Internet]. 2015 [acceso: 20 de mayo del 2015]; [aprox. 300 p]. Disponible en: http://www.worldbank.org/en/publication/wdr2015. 